\title{
POLITISCHE PROBLEME TANZANIAS \\ NACH DER SOZIALISIERUNG DES MODERNEN WIRTSCHAFTSSEKTORS
}

\author{
Von Helmut Bley
}

Um über die politische Entwicklung Tanzanias seit der Arusha-Declaration (1967) und die sich anschließende Sozialisierungspolitik mit der notwendigen historischen Dimension berichten zu können, scheint es zweckmäßig, zunächst der Frage nachzugehen, welche Koalition von sozialen Kräften die Unabhängigkeit 1961 durchgesetzt hat.

Fünf wichtige Gruppen sind zu nennen: Mit dem Ende des 2. Weltkrieges erlebten die Vermarktungsgenossenschaften der Kaffee-, Baumwoll-, Mais- und Reisproduzenten einen Aufschwung, der ein neues Element in die ökonomische und politische Entwicklung Tanganyikas brachte. Durch den enormen Anstieg der Weltrohstoffpreise während des Koreakrieges wurde damit die Position jener Bauern gestärkt, die für den Weltmarkt und die städtischen Zentren produzierten ${ }^{1}$.

Die im Kolonialdienst angestellten Afrikaner, also die berühmte „educated elite“ der Lehrer und Sekretäre hatte durch ihre Angestelltenclubs ein organisatorisches Netz geschaffen, das 1954 der Tanganyika National Union (TANU) zur Verfügung stehen sollte? ${ }^{2}$.

Eine Massenbasis ergab sich durch die Verbindungen zu der Vielzahl der lokalen Protestbewegungen gegen die Auswirkungen der Kolonialherrschaft. Insbesondere organisierten sich diese Protestbewegungen gegen Maßnahmen der kolonialen Agrarpolitik in den späten vierziger und frühen fünfziger Jahren, sofern diese repressiven Charakter annahmen: Hierzu zählen insbesondere der Kampf der „Freeman of Meru“ gegen weiße Landnahme (1950)3, die Steuerstreiks der Pare (1947)4, die Proteste gegen den Zwang bei der Terrassierung der Felder als Maßnahme gegen die Bodenerosion in den Uluguru-Bergen $(1952)^{5}$ sowie die Organisation des Auswiegens der Baumwolle durch die afrikanischen Bauern im Sukumaland gegen Manipulationen des indischen Zwischenhandels.

Der Generalstreik der Arbeiter (1947) mit Schwerpunkt in der Hafenstadt Dar es Salaam schuf eine wesentliche Voraussetzung für die Politisierung all dieser oppositionellen Aktivitäten ${ }^{6}$.

Schließlich versuchte ein Teil der Häuptlinge und Großleute, sich diesen Bewegungen anzuschließen, um der Erosion der Häuptlingsautorität durch den Mißbrauch im Interesse der Kolonialverwaltung zu entgehen. Sie sahen eine Chance zum politischen UUberleben darin, ihre traditionelle Autorität in moderne politische Führungsautorität umzuwandeln, und versuchten, sich deshalb entweder an die Spitze der ländlichen Protestbewegungen zu stellen oder aber - ohnehin mit dem Er-

1 Knappen, aber besten Uberblick zur Agrarentwicklung in Tanzania gibt. J. Iliffe, Agricultural Change in Modern Tanganyika, Historical $\Lambda$ sscociation of Tanzania Paper No. 10, Dar es Salaam 1971.

2 Für die Geschichte der TANU, insbesondere der Tanganyika African Association siehe: J. Lonsdale, Some Origins of Nationalism in East Africa, Journal of African History, Vol, IX $1968 \mathrm{~S} .131-136, \mathrm{im}$ Uberblick: Jo Iliffe, The Age of Improvement and Differentiation, in: I. Kimambo and A. Temu (HGB.) A History of Tanzania, Nairobi 1969.

3 Hierzu K. Japhet und E. Seaton, The Meru Land Case, Nairobi 1967.

4 I. Kimambo, Mbiru, Popular protest in colonial Tanzania, Historical Association of Tanzania Paper No. 9, Dar es Salaam 1971.

5 L. Cliffe, Nationalism and the Reaction to enforced agricultural Change during the Colonial Period, wieder abgedruckt in: L. Cliffe und J. Saul: Socialism in Tanzania Vol 1, Dar es Salaam 1972 S. $17 \mathrm{f}$.

6 Hierfür die ausgezeichnete Studie von J. Iliffe: A History of the Dockworkers of Dar es Salaam, in

Tanzania Notes and Records, Nr. 71, 1971 Sonderheft: Dar es Salaam, City, Port and Region, S. 119-148. 
ziehungsprivileg durch Sonderschulen ausgestattet - Teil der neuen Führungselite $\mathrm{zu}$ werden ${ }^{7}$. Generell hatten sie außerdem die Chance, über den besonderen Zugang zur Landverteilung und zu Staats- und Genossenschaftskrediten Teil der Gruppe der größeren Genossenschaftsbauern zu werden.

Versuchte man, die Machtverhältnisse in dieser Koalition zu analysieren, so ergab sich theoretisch eine übermächtige Koalition von Genossenschaftsbauern, die sich auf dem Wege zum „Kulakentum“ befanden, wie Marxisten in Tanzania zu sagen pflegen, mit jenen Vertretern der modernisierten Häuptlingsgewalt. Beides wurde durch die Kolonialmacht England gefördert, die mit dem Konzept der „indirect rule“ die Häuptlingsschicht zu stützen versuchte und zugleich im Interesse der ökonomischen Nutzung der Kolonie an rationell wirtschaftenden weltmarktorientierten Großbauern interessiert war ${ }^{8}$. Außerdem ist ein großer Teil der educated elite als die urbane Sektion dieser privatwirtschaftlich orientierten Entwicklung auf dem Lande zu sehen, jeweils mit eigener Basis in den ländlichen Gebieten, vor allem aber mit eigenen Investitionschancen sowohl im urbanen wie im ländlichen Bereich.

Die exzeptionelle Stellung Tanzanias in Afrika beruht darauf, daß diese Koalition sich wider alles Erwarten bisher als der große Verlierer nach der Unabhängigkeit erwiesen hat. Sieger wurden offensichtlich jene Teile der modernen Elite unter der Führung Nyereres, die nach einem bekannten Wort des kenyanischen Politologen Mazrui „Verräter an ihrer Klasse“ wurden, d. h. eine Sozialisierungsstrategie entwickelten und in den Grenzen, die noch zu erörtern sein werden, auch durchsetzten; dies, obwohl sie die Chance hatten, selbst als Teil einer neuen Nationalbourgeoisie $\mathrm{zu}$ investieren.

Die Frage nach ihren Koalitionspartnern und nach der Basis ihrer Stärke ist schwer zu beantworten. Sie läßt sich mit der Gegenfrage einleiten, warum es zu keinem erfolgreichen Militärputsch, getragen von der Genossenschaftsbauernschaft und ihren Repräsentanten, in den Staatsorganen kam. Ein Ansatz zur Antwort ergibt sich aus der Betrachtung der noch nicht analysierten Partner der Koalition für die Unabhängigkeit.

Zweifellos war und ist die Mehrheit der tanzanischen Bauern Kleinbauern, die höchstens über den lokalen Markt an die Marktwirtschaft angeschlossen sind. Es ist dies aber auch der unartikulierteste Teil der Bevölkerung. Zu ihnen gehören die Frauen, die, um einen Eimer aus dem Flußbett zu graben, im Extremfall über $20 \mathrm{~km} \mathrm{zu}$ gehen haben, im Normalfall immer noch 2 bis $3 \mathrm{~km}$, die Feuerholz über große Strecken heranschleppen müssen, wenn Holzkohle für sie unerschwinglich ist, deren nächstes kleines Krankenhaus über $100 \mathrm{~km}$ entfernt sein kann. Es ist die Kleinbauernschaft, die mit der Unsicherheit des Regenfalls rechnen, den Busch roden und die kleinen Äcker gegen Unkraut, Wildschweine, Affen und Vögel, das Vieh gegen Löwen verteidigen müssen. Diese Bevölkerung lebt je nach Region überwiegend nur von Mais oder Kassava, Reis oder Bananen. Sie leidet dementsprechend unter ständigem Eiweißmangel und damit unter Fehlernährung9. Es ist jene Bevölkerung, bei der die Kindersterblichkeit um 14 Pro-

\footnotetext{
7 Eine gute Studie am Beispiel der Wanyamwezı: N. Miller, The Political Survival of Traditional Leadership, in: The Journal of Modern African Studies, 1968, 6,2. Wieder abgedruckt in L. Cliffe und J. Saul, Socialism in Tanzania, S. $145-153$.

8 Für Konzept und relatives Scheitern der Politik der „indirect rule“ in Tanganyika siehe: R. Austen, Northwest Tanzania under German and British Rule, New Haven 1968.

Für den Begriff "Kulaken“ im tanzanischen Kontext siehe: H. V. E. Thoden van Velzen, Staff, Kulaks and Peasants, University of Dar es Salaam, Rural Development Paper Nr. 8, 1971.

9 Eindrucksvolle Daten selbst für landwirtschaftlich entwickelte Regionen siehe: National Nutrition
} 
zent, in speziellen Regionen um 25 Prozent liegt, die Lebenserwartung im Durchschnitt um 43 Jahre (in den Extremen um 61 bzw. 28 Jahre (1967) ${ }^{10}$.

Wenn man das Ende der Kolonialherrschaft in den fünfziger Jahren - abgesehen von dem weltpolitischen Rahmen - erklären will, dann ist es wesentlich die Unfähigkeit der Kolonialregierung gewesen, diese Massen zur Produktivitätssteigerung im Interesse der Exportwirtschaft zu mobilisieren und Hoffnung auf Befriedigung der Grundbedürfnisse zu vermitteln. Demgegenüber solidarisierte sich die Nationalbewegung mit jeder Protestbewegung und erhielt damit eine Massenbasis, die zugleich die der traditionellen Häuptlinge erodierte. Dies ermöglichte schließlich eine Sozialisierungspolitik, weil sich die ländliche Bevölkerung - und die städtischen Massen - jedenfalls in wichtigen Perioden gegen eine neue ökonomische und politische Elite mobilisieren ließen ${ }^{11}$.

Vermittelt wurden die Interessen der ländlichen Bevölkerung auf zwei Wegen: 1. durch die Vertretung der lokalen Parteiführer im Zentralkommitee der TANU $(\mathrm{NEC})^{12}$, 2. durch die Tatsache, daß sich eine Koalition von regionalen Parteiführern der unterentwickelten Regionen gegen die "Cash-Crop"-Regionen bildete, also gegen die Kaffeegebiete des Kilimanjaro und Bukoba und die Baumwollgebiete am Viktoriasee ${ }^{13}$.

Eine solche Koalition war aus drei Gründen möglich:

1. Die moderne Geschichte afrikanischer Länder begann mit ihrem Anschluß an den Weltmarkt. Dies führte zu starker interner Differenzierung. Es entstanden Regionen, die für den Weltmarkt produzierten oder Nahrung für die Cash-CropProduzenten und die Städte anbauten, und jene Regionen, für die sich keine Cash-Crops fanden, die sich selbst überlassen blieben oder von der Rekrutierung der Wanderarbeiter für die Plantagengebiete und im Südosten Tanganyikas für die Bergwerke des südlichen Afrikas betroffen wurden. Dieser Prozeß der Differenzierung begann in Tanganyika ab 1850 mit dem Aufbau des Hanđelsimperiums des Sultanats von Zanzibar ${ }^{14}$.

2. Während der Kolonialherrschaft mit ihrer systematischen Pflege von exportfähigen Produkten verschärfte sich diese Differenzierung. Dabei kam es in Deutschostafrika bis 1914 und im britischen Mandatsgebiet Tanganyika bis 1961 zu einem historisch bedeutsamen Kompromiß in der wirtschaftlichen Machtverteilung. Es gelang weißen Siedlern nicht, die Exportwirtschaft zu monopolisieren. Der Widerstand der afrikanischen Bauernproduzenten im Maji-Maji-Aufstand 1904-1906 (mit insgesamt geschätzten 250000 Opfern wegen der nachfolgenden Hungersnot) veranlaßte die deutsche Kolonialverwaltung in Dar es Salaam unter Gouverneur Rechenberg und das Reichskolonialamt in Berlin unter Staatssekretär Dernburg, Bauernproduktion als geeignete Wirtschaftsform anzuerkennen. Rechenberg, 1905 Generalkonsul in Warschau, war von der Bauernbewegung in der Ersten Russischen Revolution beeindruckt und wollte einen andauernden Bauernkrieg in

Survey, abgedruckt in Auszügen in: Food and Nutrition, in: E. J. Temu und K. v. Freyhold, AgriculSurvey, abgedruckt in Auszügen in: Food and Nutrition, in: E. J.
ture in Secondary Schools, Dar es Salaam 1972, S. 116 (vervielfältigt).

$10 \mathrm{~J}$. D. Thomas, Infant Mortality in Tanzania, Research paper, Dar es Salaam 1972, Seminar des Economic Research Bureaus (vervielfältigt).

11 Am überzeugendsten J. Iliffe, Agricultural Change . . . und Age of improvement . . . a. a. O.

12 Eine der gründlichsten Studien zur inneren Struktur der TANU mit weiterführenden Literaturhinweisen: G. A. Maguire, Toward Uhuru in Tanzania, Cambridge 1969. Außerdem: H. Bienen, Party Transformation and Economic Development. Princeton 1967.

13 Siehe Kabinettslisten im Tanzania Directory $1961 \mathrm{ff}$. Akzentuiert wurde diese Entwicklung durch den Aufstieg Kawawas als Ministerpräsident als Repräsentanten des Südens und das Herausdrängen von Bomani als Vertreter der Genossenschaftsbewegung im Sukumaland.

14 Bester UUberblick für das folgende Iliff́e, Agricultural Change... Für die Rückwirkungen des ZanzibarHandels auf afrikanische Initiativen auf dem Festland: N. Bennett, Mirambo of Tanzania 1840-1884, Oxford 1971. Vgl. A. Roberts The Nyamwezi, in: Tanzania before 1900, Dar es Salaam 1968, S. $117 \mathrm{ff}$. 
der Kolonie vermeiden. Der Gegenschlag der deutschen Siedler unter Führung des Großlandwirtes v. Lindequist als Staatssekretär (1910 Entlassung Rechenbergs) wurde durch die deutsche Niederlage im Ersten Weltkrieg gestoppt. Der Kernpunkt der Mandatslösung des Völkerbundes war, die selbständige Bauernwirtschaft in Tanganyika zu ermöglichen.

3. Ein wesentlicher Faktor für die Koalitionsbildungen ergab sich aus der ethnischen und sprachlichen Struktur Tanganyikas. In keiner dieser moderneren Wirtschaftsregionen lebten Völker, die einen entscheidenden Bevölkerungsanteil ausmachten. Die z. Z. ca. 14 Millionen Tanzanier (vergleichbar mit Rumänien, Südafrika) teilen sich auf in über 120 ethnische Gruppen, allerdings 90 Prozent davon auf der Basis nahverwandter Bantu-Sprachen.

Keines der größeren Völker hatte die Möglichkeit, in Tanzania zu dominieren. Das größte Volk, die Wasukuma, zählten nach dem Zensus von 1967 ca. 1,5 Mill., das zweitgrößte, die Chagga, 0,5 Mill. ${ }^{15}$.

Wegen der frühen Verflechtung mit dem Weltmarkt hat sich aus Kisuaheli als lingua franca eine Nationalsprache entwickelt, die vor Englisch die Grundlage der politischen Kultur des Landes geworden ist ${ }^{16}$.

Damit waren Koalitionsmöglichkeiten eröffnet, die weder Sprachen- noch Hegemonialprobleme in gravierender Weise aufwarfen. Die beiden Wege, die in dieser Frage für Tanganyika nach der Unabhängigkeit offen schienen, lassen sich an den beiden Politikern exemplifizieren, die den Anspruch auf Unabhängigkeit vor den Vereinten Nationen in den fünfziger Jahren artikulierten. Der eine war König Marealle ${ }^{17}$, Oberhäuptling der Chagga, der Kaffeepflanzer am Kilimanjaro. Dieses Volk hatte seit 1928 das am wirksamsten organisierte Genossenschaftswesen, wies die höchste Alphabetisierungsrate auf und ist in Spitzenpositionen in Armee, Bürokratie und Universität aufgrund dieses Erziehungsvorsprunges überrepräsentiert. Demgegenüber ist Präsident Nyerere Sohn eines Häuptlings des kleinen Völkchens der Wazanaki am Viktoriasee ${ }^{18}$, ein auch heute noch isolierter Landesteil. Marealle verlor noch vor der Unabhängigkeit Amt und Einfluß $\beta^{19}$, bis schließlich 1963 die Häuptlinge durch Gesetz abgeschafft wurden. Nyerere stieg zu präsidialer Machtfülle auf in Koalition mit den Vertretern des unterentwickelten Südens und des Zentrums des Landes, repräsentiert durch den aus der Gewerkschaftsbewegung und der islamischen Küstenkultur hervorgegangenen Ministerpräsidenten Kawawa.

\section{Die Entstehung der Sozialisierungsstrategie}

Die Machtergreifung der TANU unter der Führung Nyereres sowie die Ausschaltung der traditionellen Führungsschicht, zumindest auf der nationalen Ebene, sagte noch nichts über die Möglichkeit eines sozialistischen Weges aus, noch weni-

15 Zensus von 1967, zusammengefaßt nach Distrikten in: Atlas of Tanzania, ed. from Ministry of Lands, Settlement and Water, Dar es Salaan, Beiblatt zu S. 13.

16 Zusammenfassung mit weiterführender Literatur: M. H. Abdulaziz, Tanzania, National Language Policy and the Rise of Swahili Political Culture, wieder abgedruckt in: Cliffe, Saul, Socialism in Tanzania, S. 155 .

17 Zum Aufstieg und Sturz Marealle's 1950-1960 siehe A. J. Temu, The Rise and Triumph of Nationalism, in I. Kimambo, A. J. Temu: A History of Tanzania, Dar es Salaam 1969, S. 197

18 Biographische Notiz zu Nyerere in: J. Nyerere, Freedom and Unity, Dar es Salaam 1966.

19 Marealle verlor seine politische Position durch eine Wahlniederlage gegen Eliufoo 1960. Die Abschaffung der Häuptlinge erfolgte durch Gesetz mit Wirkung vom 1. 1. 1963. Siehe N. Miller, The Political Survival..., S. 153. 
ger über dessen Erfolgschancen. Man kann davon ausgehen, daß es in den ersten Jahren der Unabhängigkeit keine Sozialisierungsstrategie gegeben hat. Aber es scheint, daß eine wesentliche Voraussetzung für eine derartige Entwicklung von vornherein durch die starke ländliche Basis der TANU, vor allem aber durch die prinzipiellen politischen Überlegungen Nyereres gegeben hat. Denn eines erscheint deutlich: Von vornherein kam entgegen den "normalen“ Tendenzen der sozialen Differenzierung und damit der klassenmäßigen Aufspaltung der tanzanischen Gesellschaft unter dem Druck der Weltmarktverflechtung auch das egalitäre Prinzip zur Geltung. Dies ist ohne Julius Nyerere schwer denkbar. Durch ihn kam Konsistenz in die tanzanische Politik in Richtung auf Egalität. Bereits Jahre vor Erringung der Unabhängigkeit und lange vor einer Chance, seine Politik auch durchsetzen zu können, hat er gegen eine privatwirtschaftlich orientierte Strukturpolitik in der Landfrage entschieden Front gemacht. Die Strategie der englischen Kolonialverwaltung war wie in allen Kolonien so auch in Tanganyika die Schaffung des afrikanischen kommerzialisierten Landwirtes, sofern nicht Europäersiedlung sich durchsetzte. So formulierte der zuständige britische Landwirtschaftssekretär für Tanganyika 1952:

„Ich wünsche mir, daß aus unserer bisher undifferenzierten afrikanischen Gesellschaft eine substantielle Anzahl von reichen Leuten heranwachsen kann. Sie sollen reich genug sein, um ihre Söhne in Europa erziehen lassen zu können, sie müssen sich Autos leisten können, gute Häuser usw." Sein Ziel war eine „relativ wohlhabende Schicht" als stabilisierender Faktor auch gegen die nationale Unruhe ${ }^{20}$. Ein solches Konzept entsprach auch den Interessen dieser Schicht. Die Nguu-KaffeePflanzer-Genossenschaft stellte ihrer Genossenschaftssatzung folgende Präambel voran:

„Wir gehen davon aus, daß das Ziel der Genossenschaft ist, ihren Mitgliedern $\mathrm{zu}$ ermöglichen, daß sie Farmen besitzen, ohne auf ihnen arbeiten zu müssen " 21 . Diese Satzung wurde 1954 festgelegt, eine Woche vor Gründung der TANU.

Nyerere hat gegen die auf dieses Konzept abgestellte Landpolitik der englischen Kolonialverwaltung protestiert. 1958 erklärte er zur Einführung der Verkäuflichkeit von Land: „Wenn wir zulassen, daß man Land wie einen Umhang verkaufen darf, dann bedeutet dies, daß nach nur kurzer Zeit nur einige wenige Afrikaner Land in Tanganyika besitzen werden, und alle anderen wären Pächter. Wir werden dann mit einem Problem zu tun haben, das Antagonismen geschaffen hat unter den Völkern und das zu Blutvergießen in vielen Ländern der Welt geführt hat" ${ }^{\text {22. }}$.

Eine wesentliche Voraussetzung, die eine sozialistische Strategie in Tanzania möglich machte, war somit, daß Sozialisierung weit mehr Verhinderung von großem Eigentum bedeutete als Umverteilung von Eigentum.

Indessen, abgesehen von der Nationalisierung ungenutzten Landes und der staatlichen Landkontrolle, änderte sich an diesem internen agrarischen Differenzierungsprozeß nach der Unabhängigkeit wenig ${ }^{23}$. Die Entscheidung fiel zunächst im modernen Sektor der Volkswirtschaft.

20 D. W. Malcolm (Principal Assistant Secretary) Minute 29. 7. 1952, in Iliffe, Agricultural Change. S. 37, Anm. 43.

21 Ebenda S 39.

22 Ebenda S. 38. Wiederabdruck dieses Pamphlets Mali ya Taifa von 1958 in: J. Nyerere, Freedom and Unity, Dar es Salaam 1966, S. 55: sowohl mit Stoßrichtung gegen "wealthy immigrants" und "rich and clever Tanganyikans".

23 Für den aktuellen Stand des Landrechtes siehe: H. J. Jacobsohn: Afrikanischer Sozialismus und Bodenrecht - das Beispiel Tanzania, VRƯ, 1973, S. $173 \mathrm{ff}$. 
In diesem Sektor ließ sich auch umverteilen, zunächst zu Lasten der Europäer, später zu Lasten der Inder. Schließlich setzte die TANU zum entscheidenden Zugriff auf die Investitionschancen afrikanischer Entrepreneurs und der Staatsfunktionäre an. Dennoch war diese Umverteilung und damit eine echte Sozialisierungsstrategie, die die Machtfragen einschloß, lange Zeit nicht der Zentralpunkt der Politik. Tanzanische Politik entwickelte sich nicht in eindeutigen Richtungen, sondern, was jetzt als Sozialisierungspolitik erscheint, war möglicherweise gar nicht so langfristig angelegt. Sie ist wesentlich auch Ausfluß der bitteren Notwendigkeit, die neue staatliche Unabhängigkeit auch in eine ökonomische Unabhängigkeit zu verwandeln.

Einige ökonomische Grundtatsachen bestimmten die Situation in den Jahren nach der Unabhängigkeit. Tanzania verkaufte den Sisal, den Kaffee und die übrigen Exportprodukte auch 1969 noch zu 89 Prozent auf dem westlichen Weltmarkt, nur 5 Prozent im Rahmen der Ostafrikanischen Wirtschaftsgemeinschaft, 6 Prozent in die sozialistischen Länder. 24 Prozent des Exportes ging nach Großbritannien, 27 Prozent in die restlichen Sterlingländer, 12 Prozent in die EG - an dieser Situation hat sich trotz der Einschränkung des Handels mit Großbritannien und der Ausweitung des Handels mit China nichts Ausschlaggebendes geändert. Bis 1971 ist das Importbild ähnlich ${ }^{24}$.

Tanzania ist nach wie vor Teil des westlichen Weltwährungssystems, was auch in den parallelen Abwertungen 1972 zur Geltung kam. Diese ökonomische Grundtatsache ist die Basis der Wirtschaftspolitik, wenn auch in der Annahme von Kapital- und Entwicklungshilfe durch eine planmäßige Streuung der Engagements und durch weitgehende Ausklammerung der Großmächte USA, Sowjetunion und England ein relativer Freiraum geschaffen wurde.

Das ausländische Kapital, vermittelt durch die englischen Großbanken, kam nicht wie erwartet, weil Tanzania als eines der ärmsten Länder der Welt, ohne bekannte Mineralbasis nicht attraktiv war, um Kapital in einem Umfang anzuziehen, der eine wesentliche Beschleunigung der Entwicklung im Lande hätte stimulieren können. Hinzu kam, daß Kapitalhilfe die neugewonnene Unabhängigkeit gefährdete. Insbesondere die Haltung der Bundesrepublik Deutschland, die in strikter Anwendung der Hallsteindoktrin politische Bedingungen an die Kapital- und Militärhilfe knüpfte, als das DDR-Konsulat von Zanzibar nach Dar es Salaam als Konsequenz der Union zwischen Tanganyika und Zanzibar übersiedelte, führte zu einer außenpolitischen Grundsatzentscheidung. Tanzania brach trotz der Beeinträchtigung des ersten Fünfjahresplanes die Annahme jeder Hilfe aus der BRD ab, um vor der Welt zu demonstrieren, daß jegliche Verknüpfung von Hilfe mit politischen Bedingungen auch bei hohem Risiko abgelehnt werden würde ${ }^{25}$.

Ergebnis dieser Entwicklungen war das Konzept der „Self Reliance“. Die eigenen Resourcen sollten soweit wie möglich zur Basis der Entwicklung gemacht werden. Doppelte Konsequenz war, daß sich die tanzanische Regierung langfristig darauf einstellte, Entwicklung auf der Basis der Resourcen einer Agrargesellschaft zu betreiben, kurzfristig, daß alle modernen Resourcen des Landes in die Hand

\footnotetext{
24 The United Republic of Tanzania, The Economic Survey and Annual-Plan 1970/71, Dar es Salaam 1970, Schaubild neben S. 5 mit Vergleichsangaben für 1962, 1969.

25 Nyerere zu dieser Frage in Principles and Development, memorandum for the June 1966 meeting of the National Executive Committee of TANU, in J. Nyerere, Freedom and Socialism, Dar es Salaam 1968, $\mathrm{S} 190 \mathrm{f} .:$ "And in fact this lesson has not been altogether lost on other states which have dealings with us; there is no longer any misapprehension about our sincerity when we say we want aid without strings to our political or economic policies."
} 
der Regierung gehörten. Die Kontrolle der Außenwirtschaftsbeziehungen war die Konsequenz dieses Konzeptes. Es fielen nach der Arusha-Deklaration die Entscheidungen über die Verstaatlichung der Banken, die Durchsetzung des Prinzips der staatlichung der meisten Plantagen sowie in gewissem Abstand (1970) die Verstaatlichung des Außenhandels sowie damit verbunden eine sich stets verchärfende Devisenkontrolle.

Der ökonomische Sinn dieser Maßnahmen ergab sich aus der Ungleichgewichtigkeit der internationalen Machtverhältnisse auf dem Welt- und Geldmarkt. Die Kontrolle aller Resourcen gegenüber der Außenwelt war an sich auch noch keine sozialistische Strategie.

Wenn sie dies dennoch wurde - wenn der ökonomische Nationalismus nicht die einzige Zielsetzung blieb, sondern mit Hilfe der Kontrolle der Außenwirtschaft und der Industriepolitik auch das egalitäre Prinzip umgesetzt wurde, so war dies eine neue politische Entscheidung. Als Hebel wurde angesetzt, daß sich die Außenwirtschaftspolitik und die Verstaatlichung des modernen Sektors in ein Kampfinstrument auch gegen die Investitions- und Konsuminteressen der neuen Elite umschmieden ließ26; oder genauer, die Elite wurde mit der Hinwendung zum ökonomischen Nationalismus, die auch ihren Interessen entsprach, in das neue Stadium hinübergedrängt, fest eingespannt in das Korsett des Einparteiensystems mit Elementen einer präsidialen Diktatur. Opposition in diesen Fragen nahm den Charakter eines "silent class struggles“ an, wie es die von der Macht ausgeschlossenen leninistischen und maoistischen Theoretiker der TANU YOUTH LEAGUE und der in- und ausländischen Universitäts-Intelligenz $\mathrm{zu}$ analysieren pflegen ${ }^{27}$; d. h., Opposition innerhalb des Systems.

Die Voraussetzung dafür war die Ausbildung des Einparteienstaates. Wahlsiege von 90 Prozent für die TANU als Anerkennung für den Sieg im Kampf um die Unabhängigkeit lassen die Ausbildung des Einparteiensystems fast unvermeidlich erscheinen. Die Ausbalancierung von Zentralismus im Parteiapparat - das Werk Oskar Kambonas - und der Offnung nach unten durch die Vertretung der Regionen im NEC und durch ein Wahlrecht, daß die Konkurrenz von zwei Kandidaten der TANU in einem Wahlkreis zur Pflicht machte, versetzte sowohl die Führungsspitze in die Lage, zentralistisch Strategien durchzusetzen, ließ aber auch den Druck der Landgebiete wirksam werden. Minister und Staatssekretäre hatten und haben das Risiko zu tragen, bei Vernachlässigung ihres Wahlkreises nicht wiedergewählt zu werden.

Mit Beginn einer deklarierten sozialistischen Politik verfestigte sich dieses System. Nicht nur war verfassungsrechtlich Politik außerhalb der TANU untersagt, sondern je länger die denkbare Alternative bei einem Machtwechsel zwischen sozialistischem und privatwirtschaftlichem System wurde, blieb der Machtwechsel nur noch als Putsch oder Revolution denkbar. Soziale Systeme lassen sich nicht im Rhythmus der Wahlen auswechseln.

26 Das ist die zentrale Funktion des Leadership Codes der Arusha Declaration, so unvollständig er zunächst auch durchgesetzt wurde. Siehe L. Cliffe, Personal or Class Interest: Tanzanias Leadership Conditions in Cliffe, Socialism ...., S. $241 \mathrm{ff}$

27 Für diese außerordentlich kontroverse Debatte siehe: G. Shivji, Tanzania - The silent class struggle, zunächst veröffentlicht in "Cheche" (Ubersetzung von "Iskra“), dann als paper der Universities of East Africa Social Science Conference, Dez. 1970.

Kommentar von T. Szentes (Ungarn): Status quo and Socialism, Comments on Shivji's Paper, Tanzania, The silent class struggle. In „Maji Maji“ ${ }^{\circ}, 1971$, Nr. 1.

"Maji Maji“ ist das Nachfolgeorgan von Cheche, nachdem wegen Propagierung der leninistischen Kader"partei durch die USARF, einer außerhalb der TANU stehenden studentischen Vereinigung Organ und partei durch die USARF, einer außerhalb der TANU stehenden studentischen Vereinigung, Organ und Von tanzanisch nationalistischer Position: J. F. Rweymamu: The Silent Class Struggle in Retrospect, Paper 1972, vervielfältigi 1972, Department of Political Schience, University Dar es Salaam. 
Mit Erklärung der Sozialisierungsstrategie wurde das Verbot für Funktionäre in Partei und Staat verknüpft, privatwirtschaftlich tätig zu sein. Der Anspruch wurde erhoben, daß Leader nicht mehr als ein Haus besitzen sollten, die Anstellung von Lohnabhängigen wurde untersagt, demonstrativer Konsum wurde angeprangert. Devisen- und Importkontrolle haben im Laufe der Jahre diesen Zwang zum Konsumverzicht insbesondere ab 1971 auf die gesamte Nation, praktisch auf die schmale Schicht derer mit ausreichendem Geldeinkommen ausgeweitet.

\section{Tanzania nach der Sozialisierung des modernen Sektors}

Um die Situation in Tanzania nach Durchsetzen der Sozialisierungsmaßnahmen $\mathrm{zu}$ verstehen, ist es zweckmäßig, die Frage nach der Machtverteilung und den nunmehr vorhandenen Koalitionen erneut zu stellen ${ }^{28}$.

Die Machtblöcke, die sich auch im innerparteilichen Proporz der Schlüsselpositionen widerspiegelten, stellten sich seit 1970 wie folgt dar:

Der bäuerliche Genossenschaftsflügel igelte sich ein, um die Umformung von Marktgenossenschaften in Produktionsgenossenschaften und damit das Úbergreifen der Sozialisierungsstrategie auf das Land einzugrenzen. Dies richtete sich vor allem gegen die noch $\mathrm{zu}$ erörternde Ujamaabewegung, deren ideologische Begründung zum Hauptinhalt der politischen Aussage der TANU zu werden schien. Dabei versuchte die Genossenschaftsbewegung den Freiraum zu nutzen, daß sich der Genossenschaftsgedanke systemkonform mit quasisozialistischem Gehalt auffüllen ließ.

Die Bürokratie des Staatsapparates hatte in dieser Phase zwei Optionen. Sie konnte entweder die neue Entwicklung unterstützen, weil ihr damit die Verwaltung des sozialistischen Sektors zufiel, den sie auch im Interesse des ökonomischen Nationalismus bejahen konnte, oder sie konnte ihre Querverbindungen zur mittleren Bauernschaft in den Vordergrund rücken sowie ihre Konsum- und Investitionsinteressen zur Geltung bringen und im Extremfall Staatsstreichplänen nachgehen. Beide Wege sind von Teilgruppen beschritten worden.

Als dritte Gruppe, mit der Staatsbürokratie eng verflochten, etablierte sich das große Management aus dem Finanzsektor, der halbstaatlichen Industrie und des Groß- und Außenhandels, also das Management der Bank of Tanzania, der National Bank of Commerce, der National Insurance Company, der State Trading Corporation, der National Development Corporation (Industrie), der Staatsplantagen und der National Housing Corporation sowie der Transportinstitutionen, soweit sie nicht von der Ostafrikanischen Wirtschaftsgemeinschaft verwaltet wurden.

Dieses Management stand und steht vor der schwierigen Option, daß es die Aufrechterhaltung des modernen Exportsektors, die Sicherung der Wachstumsrate und die Initierung eines Industrialisierungsprozesses entweder mit Methoden durchzusetzen versucht, die die Hauptlast dieser Entwicklung auf die tanzanischen Kleinbauern legt, oder aber den viel schwierigeren Weg der sofortigen Entlastung der großen Mehrheit der Bevölkerung mit diesem Prozeß zu verbinden sucht.

28 Die folgende Analyse der Jahre 1971/72 ist verständlicherweise noch nicht durch wissenschaftliche Literatur abgedeckt. Die Materialbasis für diese politische Interpretation beruht auf Einzelnachrichten aus der Tagespresse sowie für die Zeit der Krise auf Informationsgesprächen. Der Verfasser hielt sich z. Z. der NEC-Tagung in und bei Iringa auf. Mit Ausnahme der Hinweise auf die Schlüsseldokumente lassen sich Einzelnachweise ohne Aufführung der Beispiele nicht in diesem Rahmen einarbeiten. 
Entgegen der üblichen Annahme, daß die organisierte Arbeiterschaft Träger einer Sozialisierungsstrategie sein würde, findet sich in Tanzania seit 1965 die Gewerkschaftsbewegung durch den Staat gleichgeschaltet. Mit dem offiziellen Streikverbot werden nicht nur staatliche Interessen an der Produktionssteigerung durchgesetzt, sondern es geht vorrangig darum, die Industriearbeiterschaft der städtischen Zentren daran zu hindern, ihre privilegierte Position - gemessen an den Einkommensverhältnissen der Kleinbauern - durch eine offensive Lohnpolitik auszunutzen. Die Politik des Vorranges der ländlichen Entwicklung führt zu Interessenkollisionen mit der Arbeiterschaft.

Ein in seiner Gewichtung schwer zu beurteilender Faktor sind die Kirchen Tanzanias. Die Katholische Kirche, die Protestantischen Kirchen und der Islam werden zweifellos durch eine im wesentlichen antisozialistische Führungsschicht repräsentiert, um so mehr, als insbesondere die christlichen Gemeinden auf der Finanzkraft des mittleren Bauerntums beruhen, sofern nicht die europäische Kirchenhilfe eingreift. Diese Kirchen versuchen in diesem tiefreligiösen Land zu verhindern, daß der sozialistische Weg in eine marxistisch orientierte und die Kirchengemeinden bedrohende areligiöse Gesellschaft führt. Pluralismus im Familienrecht und in der Kulturpolitik sind wesentliche Ziele, wie die stille Opposition gegen das kirchenlose Ujamaadorf. Auf dem Lande prägt sich der Konflikt in der Rivalität zwischen den alten Dorfeliten mit starker christlicher bzw. islamischer Basis und den jungen Kadern des Staates in Verwaltung, Partei, Schule und Krankenhaus aus ${ }^{29}$.

Die Dynamik der tanzanischen Entwicklung während der letzten drei Jahre läßt sich am Verhalten zweier dieser Machtblöcke und die Kontroll- und Handlungsmöglichkeiten des politischen Systems exemplifizieren.

Die zwei Hauptfragen der tanzanischen Politik waren: Würde sich das neue Management des staatswirtschaftlichen Sektors in die Politik des Vorranges der ländlichen Entwicklung einbinden lassen oder nach den ökonomischen Maßstäben des modernen Sektors handeln? Wie würden sich der Genossenschaftsflügel und die an der Schwelle zu rentabler Marktproduktion stehenden Kleinbauern gegenüber den kollektivistischen Anforderungen der Ujamaabewegung verhalten?

Beide Komplexe bedingen sich auf das engste. Die Krise der Sozialisierungspolitik, die 1971 aufbrach, kristallisierte sich zunächst an der Managementkrise der State Trading Corporation (STC) und floß spätestens am Jahresende 1971 mit den Entwicklungen auf dem Lande zusammen.

Das neue Management sah sich vor vielfältige Sachzwänge gestellt. In dem Klima eines militanten ökonomischen Nationalismus mit sozialistisch antiwestlicher Tendenz in der Propaganda mußte es im Interesse der Finanzierung der Entwicklungspolitik die Exportwirtschaft nach Westeuropa und in die USA nicht nur am Leben erhalten, sondern umstrukturieren, um die Abhängigkeit von den Monokulturen zu mildern. Dies galt insbesondere für die Jahre 1964-1972, als der Weltmarktpreis für Sisal verfiel. Die Politik der Importsubstitution unterlag auch für Manager, die sich an der Agrarstruktur und dem Entwicklungsstand Tanzanias zu orientieren versuchten dem $Z_{\text {wang, daß }}$ auf dem Weltmarkt nur die hochentwickelten Produktionsmittel der Industriestaaten zu haben waren. Dies hatte volkswirtschaftlich häufig wenig sinnvolle Auswirkungen nicht nur auf Be-

29 In dieser Betrachtung fehlt eine Analyse des Militärs. Aufgrund der Materiallage ist die Situation im Militär für Außenseiter undurchschaubar. Frühzeitig aufgedeckte Putschpläne von einzelnen Gruppen weisen auf eine uneinheitliche Orientierung hin. 
triebsgrößen und Produktionskosten, sondern vor allem auf die betriebswirtschaftlichen Faktoren wie die Arbeitsproduktivität, Organisation der Produktion und Wartung der Produktionsmittel auf der mittleren und unteren Ebene.

Diese Tendenzen einer Nichtanpassung der ökonomischen Organisation an die Situation des Landes wurde durch Verhaltensnormen des Managements verschärft. Auch sein Maßstab für Effizienz ist in Oxford am MTI und der TH Aachen entwickelt worden. Kritische Distanz gegenüber dem dort Gelernten wird erschwert durch das verständliche, aber problematische nationale Gefühl, daß das Beste gerade gut genug für die eigene Nation sei.

Da auch dieses „Beste“ zugleich das organisatorisch Größere ist, kamen KarriereInteressen an der Größe des von diesen Managern kontrollierten Apparates hinzu, sei dies nun eine überzentralisierte Behörde oder ein aufwendiges Großkrankenhaus.

Diese Tendenzen werden gesteigert durch den Zwang, daß wegen des Fehlens eines technisch administrativen Mittelbaus sowie wegen der Vielzahl der neuen Aufgaben die unmittelbare Verwaltung der Produktion ausländischen Experten überlassen bleiben muß.

Diese bringen nicht nur ähnliche Interessen, sondern die Verkaufsinteressen der europäischen Produktionsmittelhersteller zur Geltung. Probleme der Planimplementierung bei getrennten Informationskreisen der tanzanischen Führung, der ausländischen Produktionsverwaltung und der tanzanischen Produktionsdurchführung verschärfen das Effizienzproblem.

Standortvorteile für die Industrie in der Hafenstadt Dar es Salaam, der Zwang für das Management, enge Fühlung mit der politischen Führung zu halten, von der es selbst ein Teil ist, sowie die Konsuminteressen erleichtern eine unerwünschte Bevorzugung der städtischen Zentren.

Objektiv entstehen Gegentendenzen zur offiziellen Politik, da die Abhängigkeit vom Ausland eher gesteigert als abgebaut wird, das innerbetriebliche Verhalten vom Effiziendruck und nicht von sozialistischen Formen der Kooperation geprägt wird und das Sozialprodukt insbesondere durch Fehlplanungen leichter der Stadt zufließt und dem Lande entzogen wird.

Zudem hatte das Tempo der Sozialisierung zwar mobilisierend gewirkt, jedoch die relativ kleine Spitzengruppe im Management überfordert. Diese Aussage gilt auch dann, wenn mit Bewunderung die Qualität der tanzanischen Spitzenmanager konstatiert werden kann. Positionen in den Finanzinstituten sind mit Managern wie Jamal, Mtei, Msuya, Nsekela besetzt, die sicherlich zur internationalen Spitzengruppe gehören.

Politisch relevant wurden diese Fragen, als sich die hohen Erwartungen, die mit der Nationalisierung des Außen- und Großhandels verknüpft waren, nicht erfüllten. Überzentralisierung, Branchenunkenntnis, falsche Vorratspolitik und Schwächen im Verteilungssystem verursachten Versorgungskrisen, die die Bevölkerung in Stadt und Land gegen das politische System aufzubringen drohten.

$\mathrm{Daß}$ diese Managementkrise sich zur Systemkrise auszuweiten drohte, ergab sich daraus, daß die Versorgungskrise auch das Land traf, und zwar zu einem Zeitpunkt, als sich die marktorientierte Bauernschaft durch die Offensive in der Ujamaapolitik,

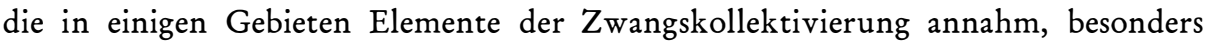
bedroht fühlte. Hinzu kam, daß der Elan der Ujamaabewegung selbst durch Ausbleiben ausreichender Produktionserfolge abbröckelte und Enttäuschte schuf. 
Unmut an der Gesamtentwicklung und an der Versorgungskrise begannen zusammenzufließen, ohne daß Gegenkräfte auf dem Lande bereit waren.

Zur Darstellung dieser Krise ist es notwendig, die Grundzüge der oft falsch eingeschätzten oder überschätzten Ujamaapolitik und die Lage auf dem Lande zu skizzieren.

Es war schon das ungelöste Problem der Kolonialverwaltung gewesen, die Masse der Kleinbauern für effizientere Produktionsformen zu mobilisieren ${ }^{30}$. Auf Anraten der Weltbank hatte die tanzanische Regierung nach der Unabhängigkeit ein Konzept der „Settlement Schemes“, d. h. der Dorfbildung betrieben, um ein erhöhtes Angebot von sozialen Diensten zu ermöglichen und Ansatzpunkte für verbesserte Agrarmethoden $\mathrm{zu}$ finden. Wie fast alle Projekte von Zentraldorfbildung, scheiterte auch das Settlement Scheme an zu hohen Investitionskosten für die einzelnen Bauern, an unzureichenden Vermarktungsmöglichkeiten und an den von den Bauern häufig mit Recht für den sicheren Ernteertrag auch in schlechten Jahren als zu risikoreich abgelehnten neuen Anbaumethoden. Insbesondere gelang die angestrebte Ausstrahlung auf das Umland nicht. Viele Settlements blieben, wenn sie existenzfähig waren, Entwicklungsinseln.

Die Arusha Declaration von 1967, die eine Politik der "Self Reliance“ formulierte, drückte auch das Eingeständnis des Scheiterns der Settlement-Politik aus. Nicht nur die Industrialisierungsstrategie Tanzanias mußte davon ausgehen, daß nicht genügend Auslandskapital zur Verfügung stehen würde, so daß kapitalintensive Modernisierungsmethoden, auch solche weit unter der Schwelle der Motorisierung, nicht gelingen würden.

Die Ujamaa-Politik der TANU31 ging einerseits davon aus, daß die Wohlstandserwartungen der Bevölkerung nicht in den Städten, sondern nur auf dem Lande selbst erfüllt werden könnten, andererseits, daß kollektive Produktionsformen mit geringerem Kapitalaufwand die notwendige Produktivitätssteigerung auf dem Lande würden erreichen können. Die Nähe zur traditionellen kommunalen Kooperation, so nahm Nyerere an, könnte die Masse der nur marginal für den Markt produzierenden Kleinbauern zur Verbesserung der Produktionsmethoden veranlassen. Elemente der Settlement-Politik blieben erhalten. Die ländliche Bevölkerung der unterentwickelten Regionen sollte eine unmittelbare Verbesserung der Lebensbedingungen erfahren. Schwerpunkte lagen in der Trinkwasser- und der Schulund Gesundheitsversorgung. Der ältere Gedanke der Produktivitätssteigerung floß mit dem Agrarprogramm Nyereres zusammen, den sozialen Differenzierungsprozeß nach Großbauern, Landarbeitern und Pächtern abzuriegeln.

Das Element des Angebotes sozialer Dienstleistungen erhielt praktisch Vorrang. Die entwickelten Produktionsgebiete und damit große Teile der Genossenschaftsbauernschaft wurden aus politischen und ökonomischen Gründen umgangen. Ujamaapolitik wurde wesentlich zu einer staatlichen Sozialpolitik auf dem Lande und in gewisser Hinsicht "Arme-Leute-Politik“.

Ujamaa geriet damit in eine Krise. $\mathrm{Da}$ die besten Produktionsgebiete ausgeklammert blieben, gelang schon wegen der ungünstigen ökologischen Umstände die Produktionssteigerung in der Regel nicht.

$\mathrm{Da}$ aber die Ujamaabewegung zur zentralen politischen Leitidee der TANU wurde, was zur Bevorzugung der Ujamaagründungen bei der Vergabe der staatlichen

30 Siehe Literaturhinweise in Anm. 5.

31 Grundlegend für das Konzept Nyereres: Socialism and Rural Development, Sept. 1967, abgedruckt in Freedom and Socialism, S. $337 \mathrm{ff}$. 
Mittel führte, siedelten sich unter dem Namen sehr unterschiedliche Dorfformen an. Es finden sich staatlich besonders beachtete Musterdörfer, von mittleren Bauern und ländlichen Händlern organisierte Zentraldörfer, vollkollektivierte Dörfer mit erfolgreicher Vermarktung und erster Verarbeitung, Dörfer mit Teilkollektivierung, bei denen die privaten Felder ungleich besser bewirtschaftet sind als das kommunale Land, Dörfer, die durch Umschließung mittelbäuerlichen Besitzes Landerwerb anstreben ${ }^{32}$.

Die Heterogenität der Dörfer ließ vieles zur Fiktion werden. Staatliche Sozialpolitik weckte eher Erwartungen als Motivationen zur Selbsthilfe, jedenfalls, wenn die Erwartungen in Vermarktungschancen oder die Überlegenheit der neuen Produktionsform enttäuscht wurden.

Wegen der ideologischen Schlüsselstellung des Ujamaakonzepts schlug bei TANU und Regierung die um sich greifende Enttäuschung über die Wirksamkeit in eine Offensivhaltung um. Einerseits wurden Forderungen nach Einbeziehung entwickelter Produktionsgebiete lautstark formuliert - in der Regel wurde das Zögern der Chagga angegriffen, andererseits große Kampagnen zur Umsiedlung in den unterentwickeltsten Regionen in die Wege geleitet. Weitgehend durchgeführt wurde die "Operation Dodoma“, von der ca. 300000 Menschen in einem der trockensten Gebiete erfaßt wurden - mit unvermeidlichen Mängeln in der Planung und Durchführung eines so komplexen Projektes, zudem unter ökologisch ungünstigen Umständen.

Politisch entscheidend für das Ujamaakonzept wurde die Offensive unter der Führung des Regional Commissioners Dr. Klerru in Iringa. Zum ersten Mal wurde ein marktorientiertes produktives Anbaugebiet Ziel der Bewegung.

Im Ismani-Tal bei Iringa hatten 1949 Lastwagenfahrer, Ladenbesitzer und Mechaniker aus Iringa, angeregt durch hohe Maispreise, Land in größerem Stil aufgekauft und mit Lohnarbeitern urbar gemacht sowie durch abgeleitete Gebirgsbäche bewässert. Betriebe von mehreren hundert Hektar Größe waren entstanden. Klerru griff diesen Großgrundbesitz in zweifacherweise an: erstens durch Landverteilung an die Landarbeiter, schließlich durch Enteignung der großen Betriebe, die auf wenige Hektar Größe reduziert wurden. Die Antwort auf diesen Angriff, der zudem von Zwangsmaßnahmen für die kommunale Arbeit auf den Ujamaadörfern begleitet war und nach Berichten aus der Umgegend auch Züge intellektueller Arroganz und Ungeduld gegenüber der ländlichen Bevölkerung zeigte, war die Erschießung Klerru's am 25. Dezember 1971. Der Täter fuhr die Leiche des Regional-Commissioner mit dem Kraftwagen zur Polizeistation und bekannte sich damit demonstrativ zu diesem politischen Mord.

Wie das Verhalten der tanzanischen Führung erkennen ließ, war die Signalwirkung, die von dieser Widerstandsmaßnahme ausging, von großer Bedeutung. In ihr kulminierte die ländliche Unruhe, die von der Ujamaabewegung und der Versorgungskrise ausgelöst war.

Methoden und Richtung der Gegenmaßnahmen der tanzanischen Regierung und der TANU sind kennzeichnend für die Machtverhältnisse und den politischen Stil nach der Sozialisierung.

32 Eine hervorragende Detailstudie hierzu: M. v. Freyhold und andere: The Potential for Ujamaa in Handeni, Seminar-paper des Department of Sociology, University of Dar es Salaam 1972. Weitere Literaturhinweise zum Ujamaaproblem: T. Möller, Tanzania: Ujamaa und Entwicklungsplanung - augewählte neuere Literatur. Herausgegeben vom Deutschen Institut für Afrika-Forschung Hamburg, erschienen in: Dokumentationsdienst Afrika, Bd. 4, Hamburg 1972. 
Während des ganzen Jahres 1971, d. h., vor der Erschießung Klerru's wurde die ländliche Unruhe offensiv bekämpft. Einerseits wurde, wie skizziert, die Ujamaabewegung beschleunigt vorangetrieben, andererseits der Schwerpunkt in der Korrektur der Politik auf die intensive öffentliche Diskussion um die STC-Krise und die Stellung des neuen Managements gelenkt.

Wichtiger Anstoß zu einer militant sozialistischen Offensive wurde ein außenpolitisches Ereignis. Mit dem Militärputsch Amin's in Uganda gegen das Regime Obote schien, so läßt sich interpretieren, nach dem Urteil entscheidender Kräfte im NEC unter der Führung Großbritanniens und Israels die ökonomische und militärische Isolierung und Einkreisung des sozialistischen Unruheherdes im Januar 1971 der Vollendung entgegenzugehen.

Auf dem Höhepunkt der Versorgungskrise ging die staats- und planwirtschaftliche Mehrheit der weniger entwickelten Staaten Uganda und Tanzania gegen Kenya innerhalb der ostafrikanischen Wirtschaftsgemeinschaft durch Umkehrung der Allianzen verloren. Der erste Grenzkonflikt zwang die TANU zur Reaktion. Die überraschende Antwort auf eine außenpolitische Bedrohung und einen Grenzkrieg war eine Wendung nach innen. Anscheinend mit beeinflußt von den 1970 in Hochverratsprozessen aufgedeckten irrealen Staatsstreichplänen Oskar Kambona's, die dieser im Londoner Exil vorbereitet hatte ${ }^{33}$, erfolgte ein Vorstoß zur Massenmobilisierung innerhalb der TANU, der auf Erschütterung der Hierarchien in Staats- und Wirtschaftsverwaltung angelegt war, die Frage des Primats der Partei gegenüber den staatswirtschaftlichen Betrieben in den Vordergrund stellte und das Recht der Basis zu spontaner Mitbestimmung in allen Institutionen verlangte.

In einer tolerierten öffentlichen Debatte in Staats- und Parteipresse und im Staatsrundfunk wurde die Kritik an der Versorgungskrise unter egalitärem Vorzeichen gegen die Technokraten in Staat und Wirtschaft umgeleitet. Parallel dazu ging die Kampagne zum Konsumverzicht, die, obwohl mitbestimmt durch die Devisenlücke, den Leadership Code der Arusha Declaration nicht nur rigoroser durchsetzte, sondern durch das Instrument der Devisenkontrolle auf alle am Konsum ausländischer Waren Beteiligten ausweitete. Das Autoimportverbot für Privatwagen wurde ausgesprochen, die Gründung von Konsumläden als Konkurrenz gegen den indischen Kleinhandel eingesetzt, Schlachtereien wurden verstaatlicht, und die Hausenteignung für Häuser im Wert von über 100000 Shillingen wurde ausgesprochen, eine Maßnahme, die zunächst den städtischen Hausbesitz der indischen Minderheit traf und dementsprechend Rückhalt in Tanzania fand. Im zweiten Durchgriff erfaßte sie aber auch Doppelbesitz und Miethäuser von Afrikanern. Schließlich wurde die STC dezentralisiert, Spitzenmanager und Minister aus dem Bereich der Indistrialisierungs- und Entwicklungspolitik ausgewechselt.

„Mongozo“, die neuen Parteirichtlinien, führten $\mathrm{zu}$ spontanen Initiativen vor allem in den Städten, zu Streiks der Arbeiter gegen mißliebiges Management in den Betrieben, zu Tätlichkeiten gegen nachlässige und unhöfliche Busfahrer im überlasteten Stadtverkehr Dar es Salaams. Die Studentenschaft der Universität provozierte den Konflikt mit dem autoritären Regiment des ehemaligen Parteisekretärs Msekwa als Vizekanzler.

33 Prozesse gegen Tibi und andere, siehe Tanzania Standard während des gesamten Jahres 1970. 
Der Armee wurde eine Volksmiliz vor allem in den Betrieben entgegengesetzt, wenn auch bei formal positiver Kooperation, da der Armee die Ausbildung der Miliz überlassen blieb.

Zwar wurde bald ein großer Teil der propagierten Spontanität mit dem Verweis auf die "official channels" wieder eingeschränkt, es gelang aber dennoch die Mobilisierung der Massen und der Partei gegen mächtige Institutionen der Staatswirtschaft. Die Kampagne gibt sicher ein Lehrstück für die Dynamisierung in einem Einparteiensystem mit einer Zentralverwaltungswirtschaft.

Sowohl Dezentralisierung der STC wie auch die von amerikanischen Unternehmungsberatern empfohlene Dezentralisation der Entwicklungspolitik milderten die ländliche Unruhe, beseitigten sie jedoch nicht. Die Ermordung Klerrus zeigte nach einem Jahr sozialistischer Offensive an, daß die Zeichen auf dem Lande weiter auf Sturm standen.

Die TANU mußte sich zu der Bedrohung äußern, die Kleinbauern, Genossenschaftsbewegung und Mittel- und Großbauern in der Ujamaabewegung und in der auch finanziellen Diskriminierung der privatwirtschaftlichen bäuerlichen Produktionsform sahen. Demonstrativ tagte das NEC in Iringa Anfang April 1972 ${ }^{34}$. Es wurden neue Leitlinien zur Landwirtschaftspolitik verkündet, die als IringaDeclaration in die offizelle Terminologie eingingen. In ihr gab Nyerere vor der Nation zu, daß in den zehn Jahren seit der Unabhängigkeit die Produktivität in der Landwirtschaft nicht gestiegen sei, sondern es nur gelungen sei, die Anbaufläche zu vergrößern.

Dementsprechend rückte die Effizienz in der Landwirtschaft in den Vordergrund. Das NEC sprach dem bäuerlichen Kleinproduzenten neue Würde zu. Die Familienwirtschaft sei nicht bedroht. Dem Genossenschaftswesen wie auch den Familienbetrieben wurde wieder normaler Zugang zu den Kreditmöglichkeiten und Berücksichtigung bei der Infrastrukturpolitik zugesagt. In seiner Budgetrede am 14. Juni 1973 wies Finanzminister Msuya auf die ersten Erfolge dieser neuen Landpolitik der Iringa-Declaration hin, seitdem die Agricultural Development Bank in allen Bereichen der Landwirtschaft Finanzierungshilfe gewähre ${ }^{35}$. Damit wurde, wie es scheint, zunächst der Angriff auf das privatwirtschaftliche Klein- und Mittelbauerntum in und außerhalb der Genossenschaftsbewegung durch ländlichen Widerstand gestoppt. Ujamaa blieb indessen als Ideologie und Programm erhalten: als Ideologie, die den Vorgang der ländlichen Entwicklung betonte und als Programm für die unterentwickelten Regionen. Ebenso blieb die Absage an Großßgrundbesitz und an Landwirtschaft auf der Grundlage von Landarbeiterrekrutierung erhalten.

Gerade in dieser Krise des tanzanischen Systems in den Jahren 1971-1972 zeigten sich wesentliche Kennzeichen für die politischen Grundlagen in Tanzania.

Die Erhaltung der einheitlichen Nationalbewegung blieb aus innen- wie außenpolitischen Gründen eine wesentliche Voraussetzung. Um dieser Einheit willen nahm die Führung einen Interessenpluralismus hin und war in kritischen Momenten zur Rücknahme wichtiger politischer Nahziele bereit. Dem entsprach die wiederholte Absage Nyereres bereits während des Wahlkampfes 1970 an eine leninistische Kaderpartei. Statt dessen zielte seine Politik auf die Erhaltung der bäuerlichen Massenpartei mit einer Art sozialistischem Zentrum.

34 Abgedruckt in Daily News vom 6. 4. 1972.

35 Abgedruckt in Daily News vom 15. 6. 1973. 
Die Sicherung einer schrittweisen Verbesserung der sozialen Dienste und der Industrialisierung setzten die Fortsetzung einer behutsamen Sicherung der Außenwirtschaftsbeziehungen und die Schonung des produktivsten Teils der Landwirtschaft voraus, dem wiederum wesentliche Zugeständnisse gemacht wurden, ohne diesen Bereich indessen in den Stand zu setzen, ein systemgefährdendes Eigenleben führen zu können.

Schließlich blieb das Instrument, breite Bevölkerungsschichten gegen die Eliten als Mittel der Kontrolle einzusetzen, auch in der Phase nach der Sozialisierung erhalten, nun aber gegen Machtfaktoren innerhalb des staatswirtschaftlichen Systems.

Aufrechterhalten blieb im übrigen das System der Machtbalance innerhalb von Partei und Staatsbürokratie, bei deutlichem Übergewicht des NEC. 


\section{The Right of Access to the Sea of African Land-locked Countries}

\section{By Zdenek Červenka}

The paper examines the problems of African land-locked countries both in terms of their access to the sea and of access to the wide area of communications with the outside world. The author maintains that the free access to the sea is derived from the fundamental principle of international law on sovereign equality of all states. The article focuses on the position of land-locked states of southern Africa Botswana, Lesotho, Swaziland and Zambia - complicated by their involvement in the confrontation of independent Africa with the colonial regime of Portugal and the white supremacy of South Africa and Rhodesia. Finally, the article deals with the role of the OAU and the author suggests a convention governing free access to the sea of African land-locked countries under OAU auspices.

\section{Political Problems of Tanzania after Socialist Transformation of the Modern Sector of the Economy}

\section{By Helmut Bley}

The essay offers an interpretation of the causes and limitations of the Tanzanian socialist strategy since 1967, especially during the years 1970-1972. The paper is based on recent published and unpublished research in this field as well as on personal observations of the author. It tries to assess the balance of power within the coalition of social forces which won independence in Tanzania. Early TANU is understood as a heterogenous movement which did not indicate socialist tendencies. Key elements in the unexpected socialist turn in independent Tanzania were 1. Nyerere's policy against land-accumulation introduced already in the fifties which was backed by rural mass support for TANU; 2 . the necessity to gain national control over the modern sector of the economy and the financial resources in order to strengthen the country against international economic pressure. Political opposition against this policy was contained by excluding the cooperative movement which controls part of the cash-crop sector from the socialist strategy and by offering the urban elite, which lost investment opportunities, the chances of administering the increased nationalized sector. The framework of the One-Party system was used to enforce this option. The new situation after implementing the socialist policy is understood as a balance between a cabinet dominated by representatives of the underdeveloped regions, the new managerial elite in state bureaucracy and para-statal economic institutions and TANU Executive Committee, which is still able to mobilise rural and urban mass support. The peasantry producing for the modern sector are isolating themselves as long as the rural strategy of Ujamaa does not touch them. The years 1970-1972 are regarded as a period of crisis, where important sectors of the rural community were negatively affected by the policy of state control in distribution and felt threatened by elements of the Ujamaa campaigns, which indicated a policy of collectivizing agriculture.

Rural discontent and the increasing problems in keeping the level of productivity led in June 1972 to a new agricultural policy, renewing the old compromise of leaving the cooperative movement and family production outside the socialist sector but maintaining the policy against accumulation of land and labour. 